\title{
INFECTION
}

\section{High-throughput drug screen strikes gold in the search for a new amoebiasis treatment}

Auranofin-an FDA-approved goldcontaining drug used as a rheumatoid arthritis treatment for 25 years - could be a promising new therapy for amoebiasis.

A collaboration led by Sharon Reed and James McKerrow overcame the challenge of working with the slow-growing anaerobic parasite Entamoeba histolytica to develop an automated high-throughput assay capable of screening large, diverse inhibitor libraries for amoebicidal activity.

"We solved the problems with earlier screening methods by using GasPak ${ }^{\mathrm{TM}} \mathrm{EZ}$ Anaerobe Gas Generating Pouch Systems and an ATP-bioluminescence based assay," explains Anjan Debnath, who made the breakthrough. "Anaerobic conditions were not required during robotic transfers, making this assay fully compatible with workstation-based automation."

Screening a library of 910 FDAapproved and unapproved bioactive compounds identified 11 that were

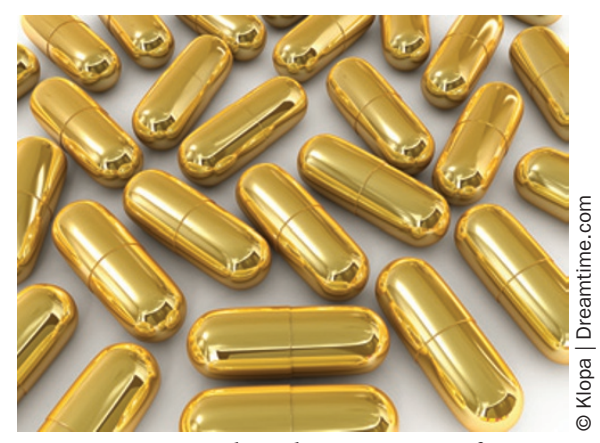

in vitro. Finally, they used a mouse model of amoebic colitis and a hamster model of amoebic liver abscess to show that the number of parasites, host inflammatory response and hepatic damage were all markedly decreased by auranofin (at doses lower than standard metronidazole treatment).

These findings prompted the FDA to give auranofin orphan-drug status as an amoebiasis treatment, so the team will now apply for clinical trial funding from the FDA and NIH. "The ability to take these findings from the laboratory to clinical trials in only a few years would not be possible if auranofin was not a 'reprofiled' drug,' concludes Reed.

Natalie J. Wood

the probable target of auranofin," says Debnath. Key enzymatic assays performed on recombinant $E h$ TrxR proved the efficacy of auranofin against the enzyme.

Reed and colleagues then confirmed the activity of auranofin in amoebae
Original article Debnath, A. et al. A high-throughput drug screen for Entamoeba histolytica identifies a new lead and target. Nat. Med. doi:10.1038/nm.2758 